\title{
Water Stress Affects Development Time but Not Takeoff Performance in the Butterfly Pararge aegeria ${ }^{\star}$
}

\author{
Simon P. Lailvaux ${ }^{1, \dagger}$ \\ Casper J. Breuker ${ }^{2}$ \\ Raoul Van Damme ${ }^{3}$ \\ ${ }^{1}$ Department of Biological Sciences, University of New \\ Orleans, 2000 Lakeshore Drive, New Orleans, Louisiana \\ 70148; ${ }^{2}$ Evolutionary Developmental Biology Research Group, \\ Department of Biological and Medical Sciences, Faculty of \\ Health and Life Sciences, Oxford Brookes University, Gipsy \\ Lane, Headington, Oxford OX3 0BP, United Kingdom; \\ ${ }^{3}$ Department of Biology, University of Antwerp, \\ Universiteitsplein 1, Wilrijk B-2620, Belgium
}

Accepted 7/27/2016; Electronically Published 11/21/2016

\begin{abstract}
Most organisms are limited in the amount and type of resources they are able to extract from the environment. The juvenile environment is particularly important in this regard, as conditions over ontogeny can influence the adult phenotype. Whole-organism performance traits, such as locomotion, are susceptible to such environmental effects, yet the specific biotic and abiotic factors driving performance plasticity have received little attention. We tested whether speckled wood Pararge aegeria L. butterflies reared under conditions of water stress exhibited poorer flight morphology and performance than control individuals. Despite large differences in mortality between treatments, we found no effects of water stress treatment on takeoff performance and only minor treatment effects on flight morphology. However, butterflies reared on water-stressed diets exhibited both significantly greater mortality and longer development times than did control individuals. Pararge aegeria larvae may compensate for this stress by prolonging development, resulting in similar realized performance capacities at least in takeoff performance in surviving adult butterflies; other measures of flight performance remain to be considered. Alternatively, the adult phenotype may be insulated from environmental effects at the larval stage in these insects.
\end{abstract}

${ }^{*}$ This paper was submitted in response to a call for papers for a Focused Issue on "Early-Life Effects on the Adult Phenotype: A Comparative Perspective." †Corresponding author; e-mail: slailvaux@gmail.com.

Physiological and Biochemical Zoology 90(1):54-62. 2017. (C) 2016 by The University of Chicago. All rights reserved. 1522-2152/2017/9001-6014\$15.00. DOI: $10.1086 / 689995$
Keywords: locomotion, development, dehydration, butterfly, whole-organism performance.

\section{Introduction}

The juvenile environment can have important effects on both the developmental trajectories and the resultant adult phenotypes of organisms (West-Eberhard 2003). The specific biotic and abiotic environmental factors driving this plasticity vary in both type and effect and include diet quality and quantity, population demography, and density, among others (Kasumovic 2013). Juvenile diet affects development time, calling effort, and longevity in the cricket Teleogryllus commodus (Hunt et al. 2004), as does the juvenile social environment, with individuals dynamically adjusting investment in certain life-history traits in response to adult male density (Kasumovic et al. 2011, 2012). In some cases, stresses in the juvenile environment can have long-term effects on the individual phenotype that persist even after the stressor has been alleviated. For example, Xiphophorus helleri fish raised in resource-limited environments experience significant locomotor costs as adults, even in adult environments with ample dietary resources (Royle et al. 2006). Understanding variation in the adult phenotype therefore requires explicit consideration of the effects of environmental factors on juvenile development. But while the effects of type and extent of variation in diet quality and quantity on phenotypic expression are increasingly well understood-particularly since the introduction of the nutritional geometric dietary framework (Simpson and Raubenheimer 1993; Raubenheimer and Simpson 2003) — other potentially important effectors of plasticity have received little attention. Dehydration, for example, has potentially serious consequences for physiology, life history, and fitness (Gatten and Clark 1989; Moore and Gatten 1989), yet effects of the juvenile hydric environment on the development and maintenance of the adult phenotype are poorly understood in most animal species.

Whole-organism performance traits (defined as any quantitative measure of how well an animal performs a dynamic, ecologically relevant task, such as jumping, flying, or biting; Bennett and Huey 1990; Lailvaux and Irschick 2006) are important phenotypic intermediaries between the organism and the environment as well as key determinants of individual fitness in a variety of ecological contexts (reviewed in Husak and Fox 2008; Irschick et al. 2008). Although performance is conceptualized primarily as a function of morphology (Arnold 1983), 
an emerging literature shows that the expression of wholeorganism performance is often plastic and thus susceptible to numerous biotic and abiotic influences (reviewed in Lailvaux and Husak 2014). Furthermore, there is an increasing appreciation that performance exists within an integrated multivariate phenotype (Ghalambor et al. 2003) and is therefore linked functionally, genetically, and developmentally with multiple other key predictors of survival and fitness (Ghalambor et al. 2004; Lailvaux et al.2010). An important challenge is therefore not only to characterize the relationships between performance and other critical fitness-related traits but also to determine how those relationships might be affected by the same environmental factors that influence performance expression and maintenance (Lailvaux and Husak 2014).

Although a handful of studies have reported effects of the juvenile environment on adult whole-organism performance in vertebrates (e.g., Garenc et al. 1999; Le Galliard et al. 2004; Royle et al. 2006; Yan et al. 2015), equivalent studies of invertebrates are scarce (however, for an example see Reaney and Knell 2015). Larval conditions in invertebrates, such as holometabolous insects, may be even more important to adult locomotor performance than in most vertebrates because the imaginal discs giving rise to adult morphological structures in insect larvae are directly affected by larval nutritional state and environment (Zera and Harshman 2001). For example, the sizes of horns and surrounding morphological structures in adult dung beetles are determined before eclosion and thus are affected by the amount and type of resources accrued during the beetle larval stage (Nijhout and Emlen 1998; Emlen 2001). Development times can also be prolonged in resourcepoor environments, in some cases to allow longer periods of compensatory feeding (Awmack and Leather 2002). If morphological structures affecting locomotion are similarly susceptible to variation in the larval environment, then those environmental conditions experienced by larvae could have long-term consequences for adult performance as well (Hughes et al. 2004). On the other hand, environmental effects on phenotype, such as that of temperature, can also be uncoupled from one stage to the next in some insects with complex life cycles (Potter et al. 2011). We currently lack a proper understanding of the developmental effects of the juvenile environment on adult whole-organism performance in holometabolous insects.

Animals face numerous challenges regarding water balance, and water availability limits both the distribution and the density of many animal species (Hawkins et al. 2003). For example, episodes of severe drought-particularly in combination with other environmental factors, such as habitat fragmentation - have significant effects on butterfly population dynamics (Oliver et al. 2015; Tack et al. 2015) and life history and morphology (Gibbs et al. 2012). Insects possess several adaptations to deal with osmotic challenges (e.g., Kestler 1985; Duncan and Byrne 2005), including an extraembryonic serosa in the egg stage (Jacobs et al. 2013; Ferguson et al. 2014). Despite studies of such adaptations, the effects of dehydration and drought on key behavioral and performance traits linked to fitness have received remarkably little attention, having been addressed only indirectly at best (Vande Velde et al. 2013).

We tested the hypothesis that larval water availability affects both development and adult flight morphology and performance in the speckled wood butterfly, Pararge aegeria L. (Nymphalidae). This organism is a well-established model system in ecology and evolution and was recently identified as one of the six drought-sensitive butterfly species in the United Kingdom that shows particularly slow recovery from repeat drought events in fragmented landscapes (Oliver et al. 2015). Flight is used in a variety of contexts in $P$. aegeria, from territorial defense in males to oviposition behavior (i.e., searching for relevant host plants) in females. Females in those drought-sensitive fragmented landscapes not only rely on flight more compared with females in woodland areas but also appear to exhibit a different wing morphology than woodland females, possibly due to plasticity (Gibbs et al. 2010). Finally, flight is energetically costly and trades off with fecundity and, possibly, immunity in female $P$. aergeria (Gibbs et al. 2010). This species is therefore an ideal organism for studying the effects of drought on flight performance as well as the potential life-history trade-offs involved.

We used a high-speed video camera to quantify takeoff performance of adult butterflies raised to maturity from caterpillars maintained on two different substrates: normally hydrated (control) and water-stressed (treatment) grasses. We measured several aspects of takeoff performance, as locomotion is a multivariate phenomenon (Lailvaux and Irschick 2006, 2007) and, hence, water-stress effects may be reflected in any of a number of performance characteristics. Specifically, we predicted that, relative to individuals reared on control grasses, individuals raised on dry, water-stressed grasses would exhibit (1) longer development times, (2) altered morphological variables related to flight performance (wing aspect ratio, thorax weight, and wing loading), and (3) compromised takeoff kinetics (velocity, acceleration, and power) and kinematics (time to peak velocity, time to peak acceleration, and time to peak power).

\section{Methods}

\section{Experimental Animals}

The butterflies were derived from an outbred laboratory stock population of Belgian Pararge aegeria butterflies and were reared under carefully controlled conditions in a growth chamber allowing for direct development (temperature day/night: $23 \%$ $18^{\circ} \mathrm{C} ; 75 \%$ humidity; $18 \mathrm{~L}: 6 \mathrm{D}$ photoperiod) on the grass species Poa trivialis. Pararge aegeria feed on grasses in nature (Shreeve 1986), and $P$. trivialis is commonly used as a laboratory food source for these butterflies. Caterpillars in the control group were reared on grass plants that had full access to water. Caterpillars in the treatment group were reared on plants that had been drought stressed and deprived of water for $30 \mathrm{~d}$ immediately prior (as in Talloen et al. 2004). (For further details on droughtstressed plant rearing using $P$. aegeria, see Gibbs et al. [2012], who used a 20-d period.) All plants had been sown on a standard soil 
substrate in plastic jars $(18 \mathrm{~cm} \times 18 \mathrm{~cm})$. The plants from both treatment groups experienced common environmental conditions, and their position was randomized every $3 \mathrm{~d}$ so as to avoid possible confounding factors due to slight but unavoidable microclimatological/environmental differences within the growth chamber. Each individual plant was enclosed in fine-mesh netting.

Four first-instar larvae were transferred to a single grass plant within $12 \mathrm{~h}$ of egg hatching. This density of same-aged caterpillars ensured a food supply without unequal competition among the caterpillars (as in Breuker et al. 2007b), thereby minimizing variability in the ability to take up resources. As a higher mortality was expected with the water-stress treatment group, 32 larvae were assigned to the control group and 72 to the treatment group. In total, 28 larvae successfully completed development in the control-diet group (87.5\% survival), but only 31 successfully developed in the low-quality-diet group (43.1\% survival). Following eclosion but before performance measurements, adult butterflies were placed in individual pots within a low-temperature $\left(10^{\circ} \mathrm{C}\right)$ growth chamber to minimize activity and given ad lib. access to a $15 \%$ sugar solution. Only animals with fully expanded wings were used in the experiment (see below). Because some time was required for the wings to properly dry and expand and because large numbers of adults sometimes emerged simultaneously, flight performance could not always be measured immediately after emergence (although in all cases we measured performance as soon as possible), and there is therefore variation in postemergence time for both diet treatments. To control for this, we recorded the time in days between emergence and measurement for each individual and included this variable as a covariate in statistical analyzes. After the performance measurements, animals were killed in a $-20^{\circ} \mathrm{C}$ freezer and dissected for morphological measurements.

\section{Flight Performance}

We measured flight performance of adult $P$. aegeria butterflies using methods similar to those of Berwaerts and Van Dyck (2004). All takeoff trials were performed in a constanttemperature room at a temperature of $29^{\circ} \mathrm{C}$, which is close to the optimal flight temperature for this species (Berwaerts and Van Dyck 2004). Individuals were kept in the temperature room for $30 \mathrm{~min}$ before performance measurement to ensure thermal equilibrium with the room temperature (Merckx et al. 2006). We placed butterflies within a small $5 \times 15 \times 15-\mathrm{cm}$ clear plastic flightway and induced them to take off from the ground up by tapping them with a pencil (following Berwaerts and Van Dyck 2004; Berwaerts et al. 2008). This chamber was large enough to allow normal behavior during takeoff (the performance stage of interest) without any danger of hitting the walls during that initial takeoff period yet narrow enough that it encouraged individuals to initiate flight forward in roughly the same direction. A high-speed Redlake camera facing the flightway in lateral view filmed each takeoff at a recording speed of 250 frames per second. We placed a mirror at a $45^{\circ}$ angle above the flightway to facilitate the simultaneous filming of both dorsal and lateral views. This provided us with two 2-dimensional flight trajectories, which we later merged into a single 3-dimensional view of each takeoff using Pythagoras's rule (Lailvaux et al. 2010, 2011). Scaling was done using $1 \times 1-\mathrm{cm}$ grids taped to the cage. To obtain maximum performance values, we filmed each individual taking off three times from a standstill with a 20 -min break between takeoffs (for justification of the use of maximal values in performance trials, see Losos et al. 2002). We then digitized each video using Didge 2.2.0. We began digitizing 20 frames before initial movement and stopped when the butterfly hit a wall or rapidly decelerated. We smoothed the $x, y$, and $z$ coordinates thus obtained using a zero-phase-shift Butterworth filter (Winter 2005) and calculated velocity and acceleration from the smoothed displacements. Mass-specific power was obtained by multiplying the observed velocity and acceleration profiles (as in Toro et al. 2003; Lailvaux et al. 2010, 2011). From these profiles, we also calculated time to peak instantaneous velocity, time to peak acceleration, and time to peak power for each takeoff as the time from initial movement of the animal until the peak values were attained for each variable. We were interested only in the initial takeoff phase in this study, and hence we did not analyze any flight data beyond the peak values for each takeoff; furthermore, because these peak values are associated with the initial power stroke of the wings and occur at the very beginning of the takeoff phase, the size of the enclosure is unlikely to affect our results, as rapid deceleration to avoid walls typically occurs long after the takeoff is complete. The flightway was also wide enough that the butterflies' wings were not impeded during takeoff, although it was narrow enough that wall effects on the takeoff stroke may exist; however, because the chamber standardized takeoff direction, these effects should apply equally to all individuals. Before each takeoff, we also measured body mass using a digital balance (MT5 Mettler). We sexed the butterflies following performance measurement to test potential interactions between treatment and sex. Consistent with general maximum performance protocols, only butterflies that yielded consistently "good" (i.e., not obviously submaximal) takeoffs were included in the final analyses (for an extensive discussion of this point, see Losos et al. 2002). Hence, two individuals from the control group and three from the treatment group that consistently exhibited clearly submaximal takeoffs were excluded from the final analyses. A further two individuals from the treatment group died immediately after eclosion and could not be measured for takeoff performance. Overall, we were able to obtain maximal takeoff measurements from 16 males and 10 females from the water-stress treatment group and 16 males and 10 females from the unstressed treatment group.

\section{Morphological Measurements}

Both fore- and hindwings were carefully removed from the thorax and placed in between two glass slides. Digital images were then taken of the ventral and dorsal wing surface with an Olympus Camedia C-3030 camera under carefully con- 
trolled light conditions. The area of each wing $\left(\mathrm{mm}^{2}\right)$ was measured using ImageJ (freely available at http://rsb.info .nih.gov/ij/), as in Breuker et al. (2007b, 2010). Measurements were done twice to assess measurement error, and regression analyses between repeated measures yielded a measurement accuracy of $98.5 \%$. The average of the first and second measurements was used in the analyses.

The thorax was dried to a constant weight at $70^{\circ} \mathrm{C}$ in a drying oven and weighed to the nearest $0.001 \mathrm{~g}$ using a Mettler digital microbalance. We calculated two important measures of insect flight morphology: (1) wing aspect ratio $(4 \times$ [wing length $\left.{ }^{2}\right] /$ total wing area; Betts and Wootton 1988) and (2) wing loading (total body weight/total wing area; Betts and Wootton 1988; Breuker et al. 2007a).

\section{Statistical Analyses}

We used the Lillifores test to verify normality in all measured variables before analysis. Mass was normalized by $\log _{10}$ transformation. We used two-way MANOVA with sex and treatment as factors and takeoff velocity, acceleration, power, time to peak velocity, time to peak acceleration, and time to peak power as dependent variables to test for differences in takeoff performance between males and females and across diet treatments. We also used two-way MANCOVA with mass as a covariate to test for such differences independent of body size. We repeated these analyses with emergence time (i.e., the time in days between eclosion and performance measurement) as a factor to test for an effect of adult age on takeoff performance. Emergence time was normalized before analysis by square root transformation.

We used MANOVA to analyze the effects of sex and treatment on flight morphology and associated factors affecting flight performance. To maximize statistical power, we carried out separate MANOVAs for each sex with thorax weight, aspect ratio, and $\log _{10}$ wing loading as dependent variables and treatment as a factor. We also carried out a separate fully factorial MANOVA to examine effects of sex and the sex $x$ treatment interaction on flight morphology. Finally, we used a generalized linear model with Poisson errors and sex and treatment as factors to test for differences in the length of larval development (i.e., the time in days from hatching to pupation) between males and females and between treatments. Generalized linear model simplification was based on the deletion test using log-likelihood ratios. All analyses were conducted using R (ver. 3.1.0; http://cran.r-project.org/).

\section{Results}

\section{Takeoff Performance}

The overall MANOVA showed no effects of treatment (Pillai's trace $=0.135, F_{6,43}=1.121, P=0.366$ ), of sex (Pillai's trace $=$ $\left.0.125, F_{6,43}=1.022, P=0.424\right)$, or of a treatment $\times$ sex interaction (Pillai's trace $=0.78, F_{6,43}=0.608, P=0.722$ ) on overall takeoff performance (comprising both the kinetic and the kinematic performance variables). Inspection of univari- ate ANOVAs revealed significant effects of sex on takeoff acceleration $\left(F_{1,48}=4.715, P<0.035\right)$ and takeoff power output $\left(F_{1,48}=4.604, P<0.037\right)$, with males exhibiting higher values than females in both cases (fig. $1 A$ ). Following size correction, the overall MANCOVA showed results similar to those of the uncorrected MANOVA for treatment (Pillai's trait $=0.131, F_{6,42}=1.052, P=0.406$ ), sex (Pillai's trace $=$ $\left.0.160, F_{6,42}=1.335, P<0.263\right)$, and treatment $\times$ sex interaction (Pillai's trace $=0.622, F_{6,42}=0.622, P=0.711$ ). The size-corrected univariate ANCOVAs for peak takeoff acceleration $\left(F_{1,47}=4.869, P<0.032\right)$ and power output $\left(F_{1,47}=4.727, P<0.035\right)$ were also significant, with values for males being larger than those for females. Emergence time had no significant effects on flight performance for either absolute (Pillai's trace $=0.92, F_{6,42}=0.708, P=0.645$ ) or size-corrected (Pillai's trace $=0.92, F_{6,41}=0.692, P=0.657$ ) data.

\section{Morphology}

The within-sex MANOVAs showed a significant treatment effect on flight morphology (i.e., wing loading and wing aspect ratio) in males (Pillai's trace $=0.290, F_{3,25}=3.409, P<0.033$ ) but not females (Pillai's trace $=0.025, F_{3,10}=0.967$ ). Specifically, the aspect ratio differed significantly between treatments in males (fig. $1 B$ ), with stressed males having significantly lower aspect ratios (and hence narrower wings) than control males $\left(F_{1,29}=5.814, P<0.023\right)$. Males and females also differed significantly in overall flight morphology (Pillai's trace $\left.=0.329, F_{3,37}=6.057, P<0.002\right)$, with males exhibiting consistently higher wing aspect ratios than females $\left(F_{1,43}=\right.$ 17.610, $P<0.001)$. However, the sex $\times$ treatment interaction was in all cases nonsignificant (Pillai's trace $=0.048, F_{3,37}=$ 0.619, $P=0.607)$.

\section{Development}

The best-fitting model for larval development time retained both sex and treatment effects (table 1; Akaike information criterion $[\mathrm{AIC}]=324.91$, no. of parameters $=2$ ), with control individuals pupating significantly sooner than droughtstressed individuals in both males and females and males exhibiting shorter development times than females (fig. 2). However, the interaction between sex and treatment (described by the next best-fitting model; AIC $=326.91$, no. of parameters $=3$ ) was not retained in the final minimum adequate model.

\section{Discussion}

The juvenile environment can have important effects on adult whole-organism performance. We predicted that restricting water availability via the host plants in Parage aegeria caterpillars would prolong larval development, alter flight morphology, and compromise flight ability in adult butterflies relative to control individuals. We found that treatment in- 


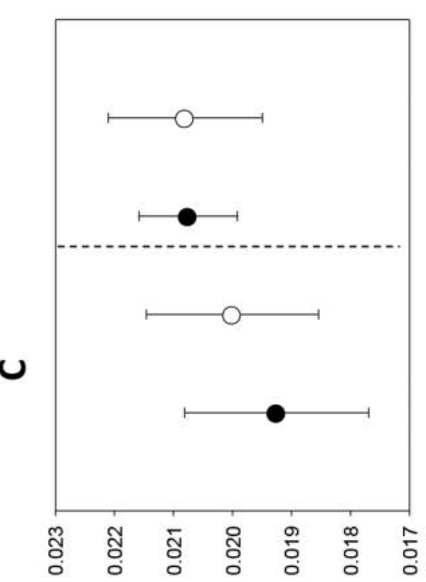

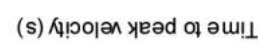
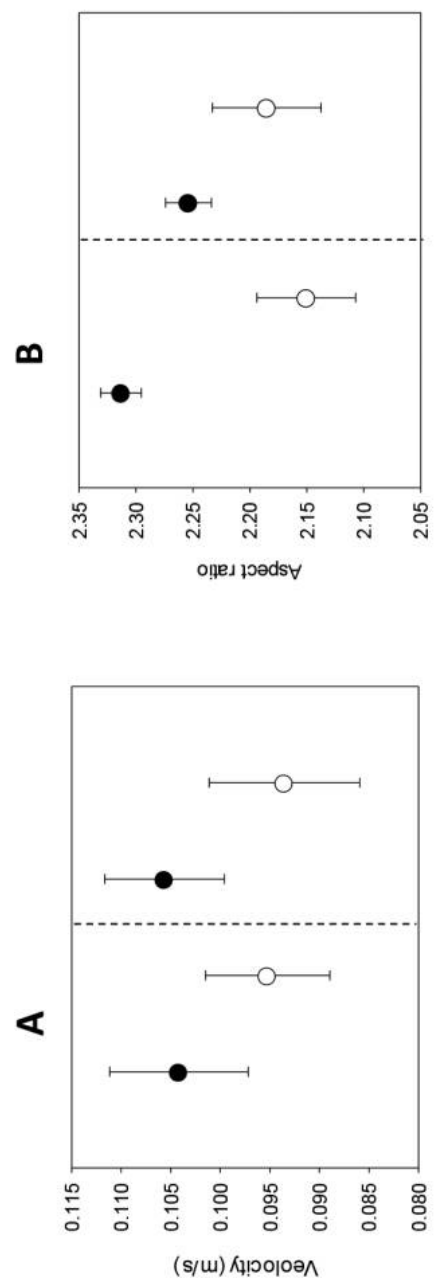

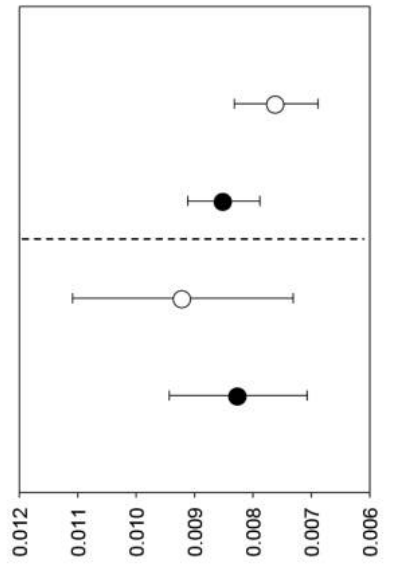

(s) uogedə|әээе yeəd 이 әயI $\perp$
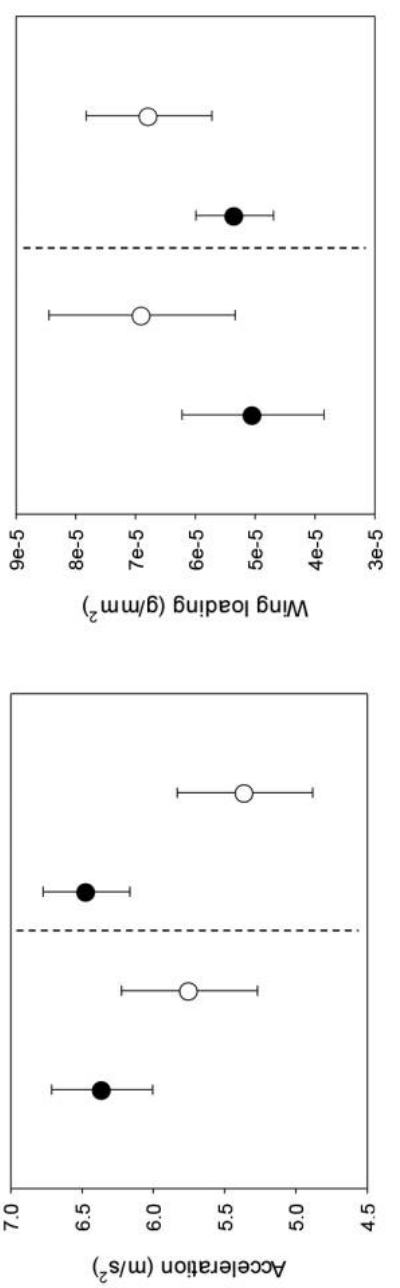

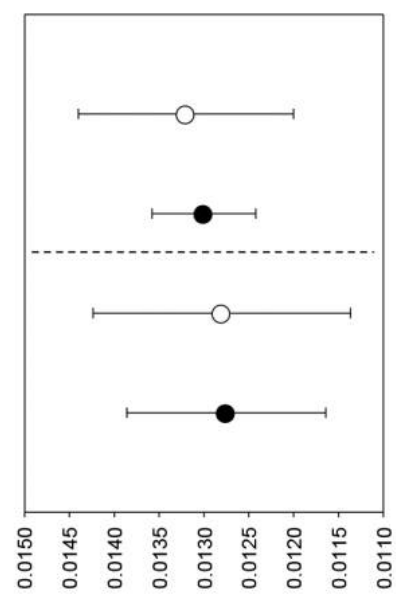

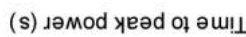

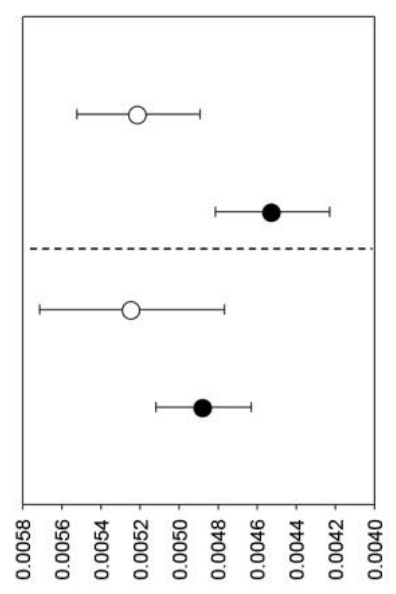

(6) ssemxesоч!

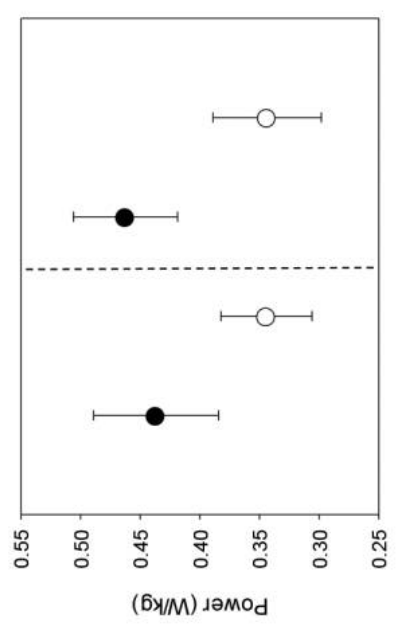


Table 1: Parameter estimates for the best-fitting generalized linear model describing the effects of sex and drought-stress treatment on development time

\begin{tabular}{lccc}
\hline Model term & Estimate & SE & $P$ \\
\hline Sex (male) & -.16 & .052 & .002 \\
Drought (treatment) & .156 & .051 & .002 \\
\hline
\end{tabular}

Note. The baseline category for sex is female, and that for drought is control. Thus, the reported values give the estimated change and associated SE in development time between the category named in the table and the baseline category.

dividuals exhibited significantly longer development times than did control individuals, supporting our first prediction (fig. 1; Gibbs et al. 2012). However, we found only partial support for our prediction of a treatment effect on flight morphology, with only male aspect ratio being significantly reduced in treatment relative to control individuals (in contrast to Gibbs et al. 2012). Despite considerably higher mortality in the treatment group relative to the control group, we found no evidence that takeoff performance in the butterfly $P$. aegeria is compromised under larval conditions of water restriction in either sex. Consequently, we are unable to reject the null hypotheses of no effect of larval water restriction via the host plant on either takeoff performance (fig. $1 A$ ) or kinematics (fig. 1C).

We focussed on the larval stage in this study because caterpillars are less mobile and thus limited in their ability to choose host food plants relative to the imago, whose mobility arguably renders them less susceptible to the effects of drought. We therefore consider it important to first understand the effects of water deprivation during the larval stadium on flight performance in isolation of the effects on the adult stadium. Although we manipulated water stress in the larval diet, we also fed adult butterflies nectar ad lib. following eclosion. Feeding load has previously been shown to have a negative effect on takeoff performance in P. aegeria (e.g., Berwaerts and Van Dyck 2004), and it may be that performance differences between treatments were therefore masked by posteclosion feedings. However, while we did not measure feeding load directly, we note that emergence time had no effect on any takeoff performance trait, and we found no significant interaction between emergence time and treatment. Takeoff performance of individuals that may have had prolonged access to nectar after emergence is therefore comparable to the performance of those that had shorter nectar access. Future studies might nonetheless consider the effects of posteclosion and adult feeding and hydration on performance explicitly, perhaps in tandem with a similar larval water-deprivation treatment.

Although we found no evidence of treatment effects on takeoff performance and kinematics, we did find large differences between treatments in larval development and survivorship. Survivorship to the final adult stage was severely compromised in the water-restricted treatment: only $43.1 \%$ of the original sample survived to emerge as adults, as opposed to $87.5 \%$ adult emergence in the normal-quality treatment, resulting in smaller sample sizes for the treatment group than expected despite allocation of more individuals to the drought-stressed treatment in anticipation of higher mortality for this group. Such mortality has also been observed in the wild, where dry spells significantly increase mortality in $P$. aegeria (Oliver et al. 2015). Of the animals that did emerge as adults, larval development times were significantly prolonged for the water-restricted treatment relative to those for the normal treatment (fig. 2). In this respect, water restriction appears to have similar results to overall dietary restriction, with previous studies of insects showing that individuals reared on poor-quality diets increase development time to allow for a longer larval feeding period, thereby compensating for low diet quality by ingesting larger quantities of food (e.g., Raubenheimer and Simpson 2003; Carvalho et al. 2005). Although one possible explanation for our results here is that the water-stressed individuals prolonged development times for similar reasons involving dietary compensation, a further possibility is that the adult phenotype is insulated from environmental effects on the larval stadium (Potter et al. 2011). Our current data set does not allow us to distinguish between these two explanations, and indeed the lack of a treatment effect on thorax weight (fig. $1 B$ ) might be considered consistent with either notion.

Although correlational studies of the link between performance and dietary quality in butterflies have, to our knowledge, never been attempted, previous studies using a correlational approach have suggested that aspect ratio is positively linked with flight capacity in butterflies. For example, Berwaerts et al. (2002) showed that aspect ratio accounted for a significant amount of variation in takeoff acceleration in $P$. aegaeria males. Here we show that although water deprivation significantly lowered aspect ratio in males (but not females), this effect did not translate into a performance difference (fig. 1) in the initial takeoff stage considered here, although it is possible that such performance effects might be manifest

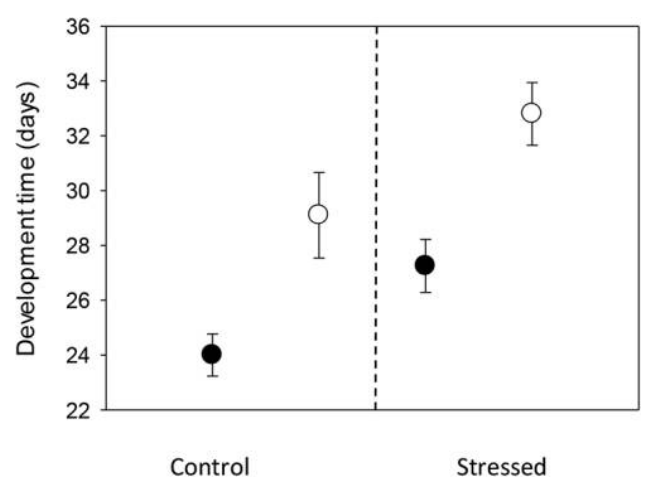

Figure 2. Larval development times for males (filled circles) and females (open circles) for control and water-stressed diet treatments. All values are means $\pm \mathrm{SE}$. 
during other locomotor contexts, such as maneuverability. In addition to the possible role played by compensation in ameliorating treatment effects, a further possibility is that the difference in aspect ratio, although statistically significant, was not large enough to translate into a biomechanical effect on flight performance. Similar mismatches between morphology and performance have been noted previously (e.g., Lauder 1996; Collar and Wainwright 2006; Lailvaux et al. 2009), and our results here suggest that flight performance in $P$. aegeria may present further scope for study of this phenomenon.

Despite our general lack of experimental support for treatment effects on takeoff performance, we did find significant effects of sex on peak takeoff acceleration and mass-specific power output, with males being better performers than females both before and after size correction. The effect of sex on acceleration is consistent with previous studies examining takeoff performance in this species (e.g., Berwaerts et al. 2002; Berwaerts and Van Dyck 2004; Berwaerts et al. 2008). Our data also show that males exhibit greater power output relative to females, a result that meshes with observed behaviors of male and female $P$. aegeria in the field. For example, whereas males are frequently observed to exhibit explosive takeoffs from rest during territory defense (Wickman and Wiklund 1983), fast takeoffs are less important to females, who rely more on sustained flight (Berwaerts et al. 2008). Thus, in addition to confirming previous reports of a sex difference in takeoff acceleration, our results for power output are consistent with observed differences in the way that males and females make use of their respective flight capacities.

Few data on the effects of water restriction on wholeorganism performance exist in any animal species, making it difficult to place our findings here within a comparative context. Locomotor capacity declines under conditions of low water availability in frogs of the genera Rana and Bufo (Gatten and Clark 1989; Moore and Gatten 1989). However, while endurance was affected by hydration in these animals, sprint speed was not, suggesting that it is the aerobic pathways supporting stamina that are more susceptible to hydration stress than anaerobically supported burst speed. Although we did not measure flight endurance in the current study, our results for burst takeoff performance are consistent with those from the frog studies in that we found no effect of drought stress on anaerobic takeoff performance. Further experimental studies would be valuable in evaluating the effect of larval drought stress, if any, on flight endurance.

\section{Acknowledgments}

We thank J. Elst for technical assistance, B. Pellegroms for establishing the initial laboratory stock population, and E. Matthysen for kindly providing the research facilities. Thanks also to S. Van Dongen, M. Gibbs, A. Herrel, and P. Aerts for valuable discussion and support. S.P.L. was supported by a postdoctoral fellowship from the Fund for Scientific Research
Flanders (FWO). Financial support for C.J.B. was obtained from research program G.0155.05 of the FWO.

\section{Literature Cited}

Arnold S.J. 1983. Morphology, performance, and function. Am Zool 23:347-361.

Awmack C.S. and S.R. Leather. 2002. Host plant quality and fecundity in herbivorous insects. Annu Rev Entomol 47: 817-844.

Bennett A.F. and R.B. Huey. 1990. Studying the evolution of physiological performance. Pp. 251-284 in D.J. Futuyma and J. Antonovics, eds. Oxford surveys in evolutionary biology. Oxford University Press, Oxford.

Berwaerts K., E. Matthysen, and H. Van Dyck. 2008. Take-off flight performance in the butterfly Pararge aegeria relative to sex and morphology: a quantitative genetic assessment. Evolution 62:2525-2533.

Berwaerts K. and H. Van Dyck. 2004. Take-off performance under optimal and suboptimal conditions in the butterfly Pararge aegeria. Oecologia 141:536-545.

Berwaerts K., H. Van Dyck, and P. Aerts. 2002. Does flight morphology relate to flight performance? an experimental test with the butterfly Pararge aegeria. Funct Ecol 16:484491.

Betts C.R. and R.J. Wootton. 1988. Wing shape and flight behavior in butterflies (Lepidoptera: Papilionoidea and Hesperioidea): a preliminary analysis. J Exp Biol 138:271-288.

Breuker C.J., P.M. Brakefield, and M. Gibbs. 2007a. The association between wing morphology and dispersal is sex-specific in the Glanville fritillary butterfly Melitaea cinxia (Lepidoptera: Nymphalidae). Eur I Entomol 104:445452.

Breuker C.J., M. Gibbs, T. Merckx, S. Van Dongen, and H. Van Dyck. 2010. The use of geometric morphometrics in studying butterfly wings in an evolutionary ecological context. In A.M.T. Elewa, ed. Morphometrics for nonmorphometricians. Lecture Notes in Earth Sciences 124. Springer, Heidelberg.

Breuker C.J., M. Gibbs, H. Van Dyck, P.M. Brakefield, C.P. Klingenberg, and S. Van Dongen. 2007b. Integration of wings and their eyespots in the speckled wood butterfly Pararge aegeria. LExp Zool 308B:454-463.

Carvalho G.B., P. Kapahi, and S. Benzer. 2005. Compensatory ingestion upon dietary restriction in Drosophila melanogaster. Nat Meth 2:813-815.

Collar D.C. and P.C. Wainwright. 2006. Discordance between morphological and mechanical diversity in the feeding mechanism of centrarchid fishes. Evolution 60:25752584.

Duncan F.D. and M.J. Byrne. 2005. The role of the mesothoracic spiracles in respiration in flighted and flightless dung beetles. LExp Biol 208:907-914.

Emlen D.J. 2001. Costs and the diversification of exaggerated animal structures. Science 291:1534-1536. 
Ferguson L., F. Marletaz, J.-M. Carter, W.R. Taylor, M. Gibbs, C.J. Breuker, and P.W.H. Holland. 2014. Ancient expansion of the Hox cluster in Lepidoptera generated four homeobox genes implicated in extra-embryonic tissue formation. PLoS Genet 10:e1004698.

Garenc C., P. Couture, M.A. Laflamme, and H. Guderley. 1999. Metabolic correlates of burst swimming capacity of juvenile and adult threespine stickleback (Gasterosteus aculeatus). L Comp Physiol B 169:113-122.

Gatten R.E. and R.M. Clark. 1989. Locomotor performance of hydrated and dehydrated frogs: recovery following exhaustive exercise. Copeia 1989:451-455.

Ghalambor C.K., D.N. Reznick, and J.A. Walker. 2004. Constraints on adaptive evolution: the functional trade-off between reproduction and fast-start swimming performance in the Trinidadian guppy (Poecilia reticulata). Am Nat 164:38-50.

Ghalambor C.K., J.A. Walker, and D.N. Reznick. 2003. Multitrait selection, adaptation, and constraints on the evolution of burst swimming performance. Integr Comp Biol 43:431438.

Gibbs M., C.J. Breuker, H. Hesketh, R. Hails, and H. Van Dyck. 2010. Maternal effects, flight versus fecundity tradeoffs, and offspring immune defence in the speckled wood butterfly, Pararge aegeria. BMC Evol Biol 10:345.

Gibbs M., H. Van Dyck, and C.J. Breuker. 2012. Development on drought-stressed host plants affects life history, flight morphology and reproductive output relative to landscape structure. Evol Appl 5:66-75.

Hawkins B.A., R. Field, H.V. Cornell, D.J. Currie, J.F. Guegan, D.M. Kaufman, J.T. Kerr, et al. 2003. Energy, water, and broad-scale geographic patterns of species richness. Ecology 84:3105-3117.

Hughes J., A. Hern, and S. Dorn. 2004. Preimaginal environment influences adult flight in Cydia molesta (Lepidoptera: Tortricidae). Environ Entomol 33:1155-1162.

Hunt J., R. Brooks, M.D. Jennions, M.J. Smith, C.L. Bentsen, and L.F. Bussieré. 2004. High-quality male field crickets invest heavily in sexual display but die young. Nature 432: 1024-1027.

Husak J.F. and S.F. Fox. 2008. Sexual selection on locomotor performance. Evol Ecol Res 10:213-228.

Irschick D.J., J.J. Meyers, J.F. Husak, and J. Le Galliard. 2008. How does selection operate on whole-organism functional performance capacities? a review and synthesis. Evol Ecol Res 10:177-196.

Jacobs C.G.C., G.L. Rezende, G.E.M. Lamers, and M. van der Zee. 2013. The extraembryonic serosa protects the insect egg against desiccation. Proc R Soc B 280:8.

Kasumovic M.M. 2013. The multidimensional consequences of the juvenile environment: towards an integrative view of the adult phenotype. Anim Behav 85:1049-1059.

Kasumovic M.M., M.D. Hall, and R.C. Brooks. 2012. The juvenile social environment introduces variation in the choice and expression of sexually selected traits. Ecol Evol 2: 1036-1047.
Kasumovic M.M., M.D. Hall, H. Try, and R.C. Brooks. 2011. The importance of listening: juvenile allocation shifts in response to acoustic cues of the social environment. LEvol Biol 24:1325-1334.

Kestler P. 1985. Respiration and respiratory water loss. Pp. 137-183 in K.H. Hoffmann, ed. Environmental physiology and biochemistry of insects. Springer London, London.

Lailvaux S.P., M.D. Hall, and R.C. Brooks. 2010. Performance is no proxy for genetic quality: trade-offs between locomotion, attractiveness, and life history in crickets. Ecology 91:1530-1537.

Lailvaux S.P. and J.F. Husak. 2014. The life-history of wholeorganism performance. $\mathrm{Q}$ Rev Biol 89:285-318.

Lailvaux S.P. and D.J. Irschick. 2006. A functional perspective on sexual selection: insights and future prospects. Anim Behav 72:263-273.

- 2007. Effects of temperature and sex on jump performance and biomechanics in the lizard Anolis carolinensis. Funct Ecol 21:534-543.

Lailvaux S.P., L.T. Reaney, and P.R.Y. Backwell. 2009. Dishonest signalling of fighting ability and multiple performance traits in the fiddler crab Uca mjoebergi. Funct Ecol 23:359-366.

Lailvaux S.P., F. Zajitschek, J. Dessman, and R. Brooks. 2011. Differential aging of bite and jump performance in virgin and mated Teleogryllus commodus crickets. Evolution 65: 3138-3147.

Lauder G. 1996. The argument from design. Pp. 55-91 in M.R. Rose and G. Lauder, eds. Adaptation. Academic Press, San Diego.

Le Galliard J., J. Clobert, and R. Ferrière. 2004. Physical performance and Darwinian fitness in lizards. Nature 432:502505.

Losos J.B., D.A. Creer, and J.A. Schulte. 2002. Cautionary comments on the measurement of maximum locomotor capabilities. L Zool (Lond) 258:57-61.

Merckx T., B. Karlsson, and H. Van Dyck. 2006. Sex- and landscape-related differences in flight ability under suboptimal temperatures in a woodland butterfly. Funct Ecol 20:436-441.

Moore F.R. and R.E. Gatten. 1989. Locomotor performance of hydrated, dehydrated, and osmotically stressed anuran amphibians. Herpetologica 45:101-110.

Nijhout H.F. and D.J. Emlen. 1998. Competition among body parts in the development and evolution of insect morphology. Proc Natl Acad Sci USA 95:3685-3689.

Oliver T.H., H.H. Marshall, M.D. Morecroft, T. Brereton, C. Prudhomme, and C. Huntingford. 2015. Interacting effects of climate change and habitat fragmentation on droughtsensitive butterflies. Nat Clim Change 5:941-945.

Potter K.A., G. Davidowitz, and H.A. Woods. 2011. Crossstage consequences of egg temperature in the insect Manduca sexta. Funct Ecol 25:548-556.

Raubenheimer D. and S.J. Simpson. 2003. Nutrient balancing in grasshoppers: behavioural and physiological correlates of dietary breadth. LExp Biol 206:1669-1681. 
Reaney L.T. and R.J. Knell. 2015. Building a beetle: how larval environment leads to adult performance in a horned beetle. PLOS ONE 10:14.

Royle N.J., J. Lindstrom, and N.B. Metcalfe. 2006. Effect of growth compensation on subsequent physical fitness in green swordtails Xiphophorus helleri. Biol Lett 2:3942.

Shreeve T.G. 1986. Egg-laying by the speckled wood butterfly (Pararge aegeria): the role of female behavior, host plant abundance and temperature. Ecol Entomol 11:229236.

Simpson S.J. and D. Raubenheimer. 1993. A multilevel analysis of feeding behavior: the geometry of nutritional decisions. Philos Trans R Soc Lond B 342:381-402.

Tack A.J.M., T. Mononen, and I. Hanski. 2015. Increasing frequency of low summer precipitation synchronizes dynamics and compromises metapopulation stability in the Glanville fritillary butterfly. Proc R Soc B 282:8.

Talloen W., H. Van Dyck, and L. Lens. 2004. The cost of melanization: butterfly wing coloration under environmental stress. Evolution 58:360-366.
Toro E., A. Herrel, B. Vanhooydonck, and D.J. Irschick. 2003. A biomechanical analysis of intra- and inter-specific scaling of jumping and morphology in Caribbean Anolis lizards. I Exp Biol 206:2641-2652.

Vande Velde L., N. Schtickzelle, and H. Van Dyck. 2013. Effect of larval food stress on male adult behaviour, morphology and reproductive investment in the butterfly Pararge aegeria. Evol Ecol 27:221-234.

West-Eberhard M.J. 2003. Developmental plasticity and evolution. Oxford University Press, New York.

Wickman P.O. and C. Wiklund. 1983. Territorial defense and its seasonal decline in the speckled wood butterfly (Pararge aegeria). Anim Behav 31:1206-1216.

Winter D.A. 2005. Biomechanics and motor control of human movement. Wiley, Hoboken, NJ.

Yan G.J., X.K. He, Z.D. Cao, and S.J. Fu. 2015. Effects of fasting and feeding on the fast-start swimming performance of southern catfish Silurus meridionalis. L Fish Biol 86:605-614.

Zera A.J. and L.G. Harshman. 2001. The physiology of lifehistory trade-offs in animals. Annu Rev Ecol Syst 32:95-126. 